\title{
Radioimmunotherapy Confers Long-Term Survival to Lymphoma Patients with Acceptable Toxicity: Registry Analysis by the International Radioimmunotherapy Network
}

\author{
Karin Hohloch ${ }^{1}$, Angelika Bischof Delaloye ${ }^{2}$, Christiane Windemuth-Kieselbach ${ }^{3}$, Jose Gómez-Codina ${ }^{4}$, \\ Werner Linkesch ${ }^{5}$, Woijciech Jurczak ${ }^{6}$, Roberto Cacchione ${ }^{7}$, Cheolwon Suh ${ }^{8}$, Pier Luigi Zinzani ${ }^{9}$, and Lorenz Trümper ${ }^{1}$ \\ ${ }^{1}$ Göttingen Comprehensive Cancer Center, Georg August University, Göttingen, Germany; ${ }^{2}$ Centre Hospitalier Universitaire Vaudois, \\ Lausanne, Switzerland; ${ }^{3}$ Alcedis GmbH, Independent CRO, Giessen, Germany; ${ }^{4}$ Hospital Univerisitario La Fe, Valencia, Spain; \\ ${ }^{5}$ Klinische Abteilung für Hämatologie, Medical University Graz, Graz, Austria; ${ }^{6}$ Department of Haematology, Jagiellonian \\ University, Cracow, Poland; ${ }^{7}$ CEMIC, Centro de Educación, Médica e Investigaciones Clínical, Norberto Quirno, Buenos Aires, \\ Argentina; ${ }^{8}$ Ulsan College of Medicine, Seoul, Korea; and ${ }^{9}$ Istituto di Ematologia e Oncologia Medica, Università di Bologna, \\ Bologna, Italy
}

The Radioimmunotherapy Network (RIT-N) is a Web-based, international registry collecting long-term observational data about radioimmunotherapy-treated patients with malignant lymphoma outside randomized clinical studies. The RIT-N collects unbiased data on treatment indications, disease stages, patients' conditions, lymphoma subtypes, and hematologic side effects of radioimmunotherapy treatment. Methods: RIT-N is located at the University of Göttingen, Germany, and collected data from 14 countries. Data were entered by investigators into a Web-based central database managed by an independent clinical research organization. Results: Patients $(1,075)$ were enrolled from December 2006 until November 2009 , and 467 patients with an observation time of at least 12 mo were included in the following analysis. Diagnoses were as follows: $58 \%$ follicular lymphoma and $42 \%$ other B-cell lymphomas. The mean overall survival was 28 mo for follicular lymphoma and 26 mo for other lymphoma subtypes. Hematotoxicity was mild for hemoglobin (World Health Organization grade II), with a median nadir of $10 \mathrm{~g} / \mathrm{dL}$, but severe (World Health Organization grade III) for platelets and leukocytes, with a median nadir of $7,000 / \mu \mathrm{L}$ and $2.2 / \mu \mathrm{L}$, respectively. Conclusion: Clinical usage of radioimmunotherapy differs from the labeled indications and can be assessed by this registry, enabling analyses of outcome and toxicity data beyond clinical trials. This analysis proves that radioimmunotherapy in follicular lymphoma and other lymphoma subtypes is a safe and efficient treatment option.

Key Words: B-cell lymphoma; radioimmunotherapy; registry; treatment; RIT-Network

J Nucl Med 2011; 52:1354-1360

DOI: 10.2967/jnumed.111.089920

\footnotetext{
Received Mar. 2, 2011; revision accepted Jun. 20, 2011.

For correspondence or reprints contact: Karin Hohloch, Department of Haematology and Oncology, Göttingen Comprehensive Cancer Center, Georg August University Göttingen, D 37099 Göttingen, Germany.

E-mail: karo.hohloch@med.uni-goettingen.de

Published online Aug. 8, 2011.

COPYRIGHT @ 2011 by the Society of Nuclear Medicine, Inc.
}

$\mathbf{R}$ adioimmunotherapy is a highly lymphoma-specific therapeutic approach with low side effects and a good tolerability established in the treatment of relapsed follicular lymphoma (FL) and in consolidation after first-line treatment (1). The current standard first-line therapy for FL patients in limited stage consists of involved-field radiation, with a local control rate of $95 \%$ and disease-free and overall survival (OS) rates of 37\%-94\% and 40\%-93\%, respectively (2). In advanced stages, however, FL is deemed incurable by conventional therapy. For asymptomatic patients with advanced-stage FL, watchful waiting is the recommended strategy, whereas for symptomatic patients a variety of single-agent or multiagent chemotherapy options with high response rates are available. The introduction of rituximab, a monoclonal anti-CD20 antibody, when combined with different chemotherapy regimens resulted in a significant superior treatment outcome, compared with chemotherapy alone $(3,4)$. Relapses after conventional therapies are frequent, and subsequent treatment approaches are usually hampered by decreased response rates and shorter durations of remission with each successive treatment $(5,6)$.

The introduction of radioimmunotherapy in 1993 provided a new and effective treatment option for FL patients. The active principle of radioimmunotherapy in lymphoma is the targeting of lymphoma cells by highly specific antibodies labeled with radioactive isotopes acting primarily by killing lymphoma cells via emission of $\beta$-particles (7). Antibodies labeled with the radioactive isotopes ${ }^{131} \mathrm{I}$ or ${ }^{90} \mathrm{Y}$ have been applied in patients with advanced or heavily pretreated B-cell lymphoma (8-12), with high response rates and promising results regarding progression-free survival (PFS) and OS. First-line treatment with ${ }^{131}$ I-tositumomab showed excellent and durable response rates (13). More recently it was shown in a randomized study that patients with FL having responded to first-line therapy and treated with 
${ }^{90}$ Y-ibritumomab tiuxetan (Zevalin; Bayer Vital GmbH) had a significantly longer PFS than controls (14).

To date, 2 radiolabeled anti-CD20 antibodies $\left({ }^{131} \mathrm{I}\right.$-tositumomab [Bexxar; GlaxoSmithKline] and ${ }^{90}$ Y-ibritumomab tiuxetan) are approved for the treatment of patients with FL. ${ }^{131}$ I-tositumomab is available in the United States, and ${ }^{90}$ Y-ibritumumab is approved in Europe, the United States, Asia, and Africa.

Only small numbers of patients treated with radioimmunotherapy are included in clinical trials; therefore, clinical data regarding efficacy and toxicity especially outside the labeled indications are missing. To gain a better insight into the clinical use of radioimmunotherapy, the RIT Network (RIT-N) was established in December 2006. The RIT-N is a Web-based, international registry that collects observational data (e.g., indication, treatment outcome, and side effects) from radioimmunotherapy-treated patients with malignant lymphoma outside clinical trials (http:// www.rit-network.net).

Here, we present the first outcome data from the RIT-N based on more than 1,000 registered patients. We confirm that radioimmunotherapy is a safe and effective treatment for patients with FL; interestingly, we show for the first time, to our knowledge, in a larger patient group that radioimmunotherapy is also efficient for patients with other lymphoma subtypes in different stages of the treatment course.

\section{MATERIALS AND METHODS}

The RIT-N evolved from different national radioimmunotherapy registries in Austria, Germany, Switzerland, and Spain. A Web-based electronic data-capturing (EDC) system was developed to be used by all participants. To share the same core elements, datasets already used in the various national registries were implemented. The core data include age, indication, lymphoma subtype, clinical course, and hematologic side effects. For the purpose of specific evaluation projects such as the one presented here, datasets from the national registries are merged with the international database. Ethics committee approval is obtained on a national basis by the respective national radioimmunotherapy registry chairs. Informed consent of patients is mandatory, mainly relating to the process of anonymized data collection and storage within the registry database. An institutional review board vote was provided by the Ethics Committee of the University of Göttingen.

The Web-based EDC system is managed by a professional clinical research organization (Alcedis $\mathrm{GmbH}$, Giessen, Germany). The clinical research organization ensures data privacy protection and quality assurance according to recognized guidelines and standards such as the Guidance on Computerised Systems Used in Clinical Trials (21 Code of Federal Regulations part 11) of the Food and Drug Administration. Access to the EDC systems follows the Principle of Least Privileges realized through a role-based access control and corresponding process rights. Access authorization to the software is granted individually by means of user accounts (user identification and password). Patients are recorded and saved anonymously in the EDC database.

At the national level, chairpersons chosen by the investigators are responsible for running the registry. Chairs can access only their respective national datasets. Analyses and publications are discussed within the steering committee, comprising the national chairpersons, on a regular basis. Results are communicated from the steering committee to all users via the national chairs. Members of the RIT-N may propose analyses and, after discussion within the steering committee, perform and publish these data.

\section{RESULTS}

\section{Patient Characteristics}

Until November 2009, 1,075 patients from 14 countries were enrolled into the registry. After registration, patients were observed and documented for a minimum of $12 \mathrm{mo}$, with the option of documentation up to 36 mo after radioimmunotherapy. To date, complete datasets for 467 patients (Fig. 1) with a follow-up of at least 12 mo are available. Only these patients are included in the following analysis. The median patient age at primary diagnosis was $59 \mathrm{y}$

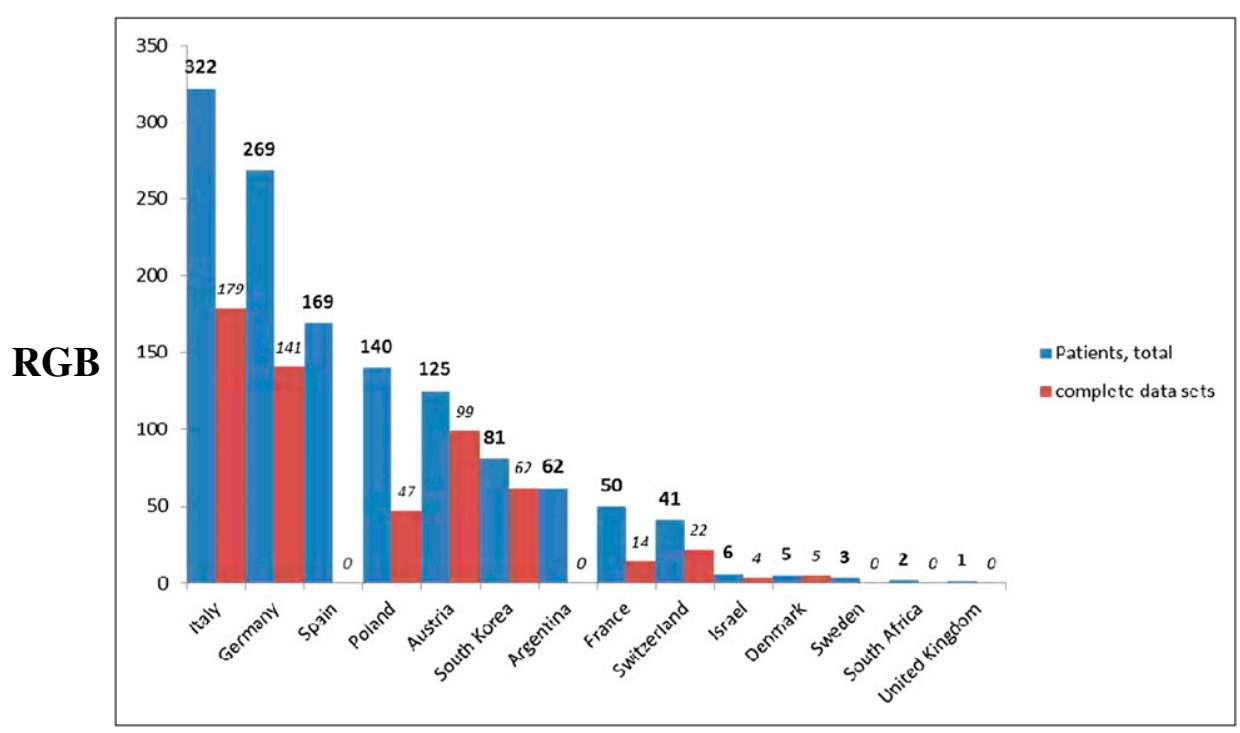

FIGURE 1. Between December 2006 and November 2009, there were 1,075 registered patients, for which 467 were complete datasets, with documentation for at least $12 \mathrm{mo}$. 
(range, 17-88 y) across countries. Forty-five percent of patients were older than $60 \mathrm{y}$ at the time of primary diagnosis (Table 1).

\section{Disease Characteristics}

Radioimmunotherapy was used to treat patients with a variety of histologic lymphoma subtypes. Lymphoma subtype was documented for all 467 patients at initial diagnosis. FL, for which radioimmunotherapy is approved, was the most common lymphoma subtype $(58 \%, n=271)$, including FL grades 1, 2, and 3a, followed by diffuse large B-cell lymphoma $(20 \%, n=94)$, mantle cell lymphoma (14\%, $n=65)$, chronic lymphatic leukemia $(5.1 \%, n=24)$, and marginal zone lymphoma $(2.8 \%, n=13)$.

TABLE 1

Patient Characteristics

\begin{tabular}{|c|c|}
\hline Characteristic & $n$ \\
\hline No. of patients & 467 \\
\hline \multicolumn{2}{|l|}{ Sex } \\
\hline Men & 250 \\
\hline Women & 217 \\
\hline \multicolumn{2}{|l|}{ Age $(y)$} \\
\hline Median & 59 \\
\hline Range & $17-88$ \\
\hline No. of patients older than $60 \mathrm{y}$ & $210(45)$ \\
\hline \multicolumn{2}{|l|}{ Lymphoma subtype } \\
\hline FL I-III a & $271(58)$ \\
\hline Diffuse large B-cell lymphoma & $94(20)$ \\
\hline Mantle cell lymphoma & $65(14)$ \\
\hline B-chronic lymphatic leukemia & $24(5.1)$ \\
\hline Marginal zone lymphoma & $13(2.8)$ \\
\hline \multicolumn{2}{|l|}{ Stage } \\
\hline 1 & $24(5.2)$ \\
\hline II & $63(13.5)$ \\
\hline III & $104(22.3)$ \\
\hline IV & $268(57.4)$ \\
\hline $\mathrm{E}$ & $4(0.9)$ \\
\hline Not determined & $4(0.9)$ \\
\hline \multicolumn{2}{|l|}{ Previous therapies } \\
\hline Chemotherapy & 445 \\
\hline $1-3$ & $342(77)$ \\
\hline $4-6$ & $89(20)$ \\
\hline$>7$ & $14(3)$ \\
\hline Radioimmunotherapy & $22(4.7)$ \\
\hline \multicolumn{2}{|c|}{ Autologous stem cell transplantation } \\
\hline 1 & $63(13.4)$ \\
\hline 2 & $3(0.6)$ \\
\hline Radiotherapy & 88 \\
\hline 1 & $67(76)$ \\
\hline 2 & $16(18)$ \\
\hline$\geq 3$ & $5(6)$ \\
\hline \multicolumn{2}{|l|}{$\begin{array}{l}\text { Bone marrow infiltration before } \\
\text { radioimmunotherapy }\end{array}$} \\
\hline No. of patients & 467 \\
\hline Infiltration & $72(15)$ \\
\hline$<20 \%$ & $59(13)$ \\
\hline$>20 \%$ & $13(3)$ \\
\hline Data in parentheses are percenta & \\
\hline
\end{tabular}

At the time of diagnosis, patients were at various disease stages; most patients had stage IV disease according to the Ann Arbor Classification. Stage at the time of radioimmunotherapy was not documented because tumor stage determination in relapse or progression of non-Hodgkin B-cell lymphoma is not regularly documented. Before radioimmunotherapy and on enrollment into the RIT-N, $15 \%(n=72)$ of patients had bone marrow infiltration. Only 13 patients had a bone marrow infiltration greater than $20 \%$ (Table 1).

\section{Indication for Radioimmunotherapy}

Patients with FL received radioimmunotherapy mainly as consolidation $(45 \%)$ after first- and second-line therapy and for recurrence $(37.7 \%)$ in second- and more than third-line therapy. Radioimmunotherapy was given to $11.8 \%$ of registered patients for refractory FL, mainly as a treatment beyond the third line.

For patients with other lymphoma subtypes, radioimmunotherapy was predominantly used as consolidation (69\%) in first-line therapy and in recurrence (13\%) in second- and more than third-line therapy (Table 2).

Regarding the remission rates (Table 3 ) and time to progression, there are no significant differences between the patients treated according to the different indications (Fig. 2).

\section{Previous Therapies}

Before radioimmunotherapy, patients received a variety of treatments. Most patients received up to 3 previous chemotherapies and up to 2 previous radiotherapies. Twentytwo patients had already received radioimmunotherapy, and 66 patients underwent stem cell transplantation before radioimmunotherapy (Table 1). Patients received radioimmunotherapy at various stages throughout the treatment pathway; $61.2 \%$ of the patients received radioimmunotherapy as a first- or second-line therapy (Fig. 3).

\section{Radioimmunotherapy}

The patients in this analysis were mainly treated with ${ }^{90}$ Y-ibritumomab tiuxetan $(n=437)$ at a median total dose of $993 \mathrm{MBq}$, equivalent to a median dose of $0.39 \mathrm{mCi} / \mathrm{kg}$ evaluable for 326 patients (range, 0.37-24.79 MBq [0.01$0.67 \mathrm{mCi} / \mathrm{kg}]$ ). Patients with $\mathrm{FL}$ received radioimmunotherapy a median of 35.1 mo after the diagnosis of lymphoma, and patients with other lymphoma subtypes, in a median time of 12.2 mo after.

\section{Outcome}

Hematotoxicity as the major side effect of radioimmunotherapy in lymphoma patients is shown in Table 4. There was no significant difference between patients with FL and patients with other lymphoma histologies. Time to complete recovery of the blood count (hemoglobin $>12 \mathrm{~g} /$ $\mathrm{dL}$, platelets $>150,000 / \mu \mathrm{L}$, and leukocytes $>4,300 / \mu \mathrm{L})$ was a median of $99 \mathrm{~d}$ for patients $(n=313)$ with FL (range, 29-1,323 d) and 97 d for patients with other lymphoma subtypes (range, 11-1,076 d). For hemoglobin, the nadir 
TABLE 2

Indication for Radioimmunotherapy

\begin{tabular}{|c|c|c|c|c|c|c|c|c|}
\hline \multirow[b]{2}{*}{ Indication } & \multicolumn{4}{|c|}{ Follicular lymphoma, line $(n=236)$} & \multicolumn{4}{|c|}{ Other lymphoma subtypes, line ( $n=172)$} \\
\hline & All & First & Second & Third through eighth & All & First & Second & Third through eighth \\
\hline Consolidation & $107(45)$ & 62 & 21 & 24 & $119(69)$ & 79 & 21 & 19 \\
\hline Conditioning & $3(1.3)$ & 0 & 2 & 1 & $5(2.9)$ & 2 & 1 & 2 \\
\hline Primary therapy & $3(1.3)$ & 3 & 0 & 0 & $5(2.9)$ & 5 & 0 & 0 \\
\hline Recurrence & $89(37.7)$ & 0 & 30 & 59 & $23(13)$ & 0 & 7 & 16 \\
\hline Therapy refractory & $28(11.8)$ & 1 & 3 & 24 & $6(3.4)$ & 0 & 1 & 5 \\
\hline Other & $6(2.5)$ & 0 & 3 & 3 & $14(8)$ & 6 & 4 & 4 \\
\hline
\end{tabular}

was reached in a median of $47.5 \mathrm{~d}$ (range, 6-406 d) for FL and $42 \mathrm{~d}$ (range, 0-418 d) for patients with other lymphoma subtypes. For platelets, the nadir was reached in a median $35 \mathrm{~d}$ (range, 6-813 d) for FL and $35 \mathrm{~d}$ (range, 7-888 d) for patients with other lymphoma subtypes. For leukocytes, the nadir was reached in a median of $40 \mathrm{~d}$ (range, 8-406 d) for FL and $38 \mathrm{~d}$ (range, 0-418 d) for patients with other lymphoma subtypes.

Response was documented for 448 patients (260 with FL and 188 with other lymphoma subtypes). Three hundred thirty-six $(75 \%)$ patients achieved a complete remission (CR), $70(16 \%)$ had a partial remission, $17(4 \%)$ had stable disease, and $20(4 \%)$ had progressive disease. For $5(1 \%)$ patients, response was not documented. There was no significant difference regarding the response rates between patients with FL and patients with other lymphoma histologies. The CR rate in FL was 73\%, as compared with $77 \%$ in other lymphoma subtypes (Table 3 ).

For patients with FL, mean OS was 28 mo (2 patients died), which is not significantly better $(P=0.23$, log-rank test) than for patients with the other lymphoma subtypes (mean OS, 26 mo [5 patients died]) (Fig. 4A). The PFS is also not different between the 2 groups, with a mean PFS of 33.6 mo (22 patients showing a progression died) for FL and a mean PFS of 25.4 mo (182 patients showing a pro- gression died) for the other lymphoma subtypes $(P=0.8$, log-rank test) (Fig. 4B).

Five patients were documented with myelodysplastic syndrome and 2 patients with an acute leukemia after having received radioimmunotherapy. One patient each with breast cancer, prostate cancer, glioblastoma multiforme, and non-small-cell lung cancer was reported as having secondary malignancies, amounting to a rate of $1.5 \%$ for hematologic malignancies and $0.8 \%$ for solid tumors.

\section{DISCUSSION}

Among patients enrolled into the RIT-N, FL was the most common lymphoma subtype. These findings may reflect the perceived suitability of radioimmunotherapy for treating FL, because this lymphoma subtype is known to be highly sensitive to radiotherapy; moreover, radioimmunotherapy is approved only for this indication (15). The median patient age $(59 \mathrm{y})$ may also be a reflection of the most common lymphoma subtype, because most patients with FL generally present at a median age of 60 y (15). Response rate and OS showed no significant differences between patients with FL and with other lymphoma subtypes. Side effects, especially hematotoxicity, were also not significantly different between lymphoma entities. In the pivotal studies with ibritumomb tiutexan for heavily pre-

TABLE 3

Response to Radioimmunotherapy

\begin{tabular}{|c|c|c|c|c|c|c|c|}
\hline \multirow[b]{2}{*}{ Response } & \multirow[b]{2}{*}{ All } & \multicolumn{3}{|c|}{$\mathrm{FL}$} & \multicolumn{3}{|c|}{ Other lymphoma } \\
\hline & & First line* & Relapse $^{\dagger}$ & No line available $\ddagger$ & First line ${ }^{*}$ & Relapse $^{\dagger}$ & No line available $\ddagger$ \\
\hline $\mathrm{CR}$ & $336(75)$ & $57(87.7)$ & $101(58.4)$ & 32 (91) & $71(84.5)$ & $58(66)$ & $17(77)$ \\
\hline Partial remission & 70 (16) & $5(7.7)$ & $36(21)$ & $1(2.9)$ & $11(13)$ & 15 (17) & $2(9)$ \\
\hline Stable disease & $17(4)$ & $2(3)$ & $10(5.8)$ & $1(2.9)$ & $0(0)$ & $2(2.3)$ & $2(9)$ \\
\hline Progressive disease & $20(4)$ & $0(0)$ & $12(7)$ & $1(2.9)$ & $0(0)$ & $6(6.8)$ & $1(4)$ \\
\hline ND & $5(1)$ & $1(1.5)$ & $14(8.1)$ & $0(0)$ & $2(2.3)$ & $7(8)$ & $10(3)$ \\
\hline \multicolumn{8}{|c|}{$\begin{array}{l}{ }^{*} \text { Consolidation first line or primary therapy. } \\
{ }^{\dagger} \text { Recurrence or therapy-refractory. } \\
\text { 苂ine of therapy was not documented. } \\
\text { ND = not done. } \\
\text { Data in parentheses are percentages. }\end{array}$} \\
\hline
\end{tabular}




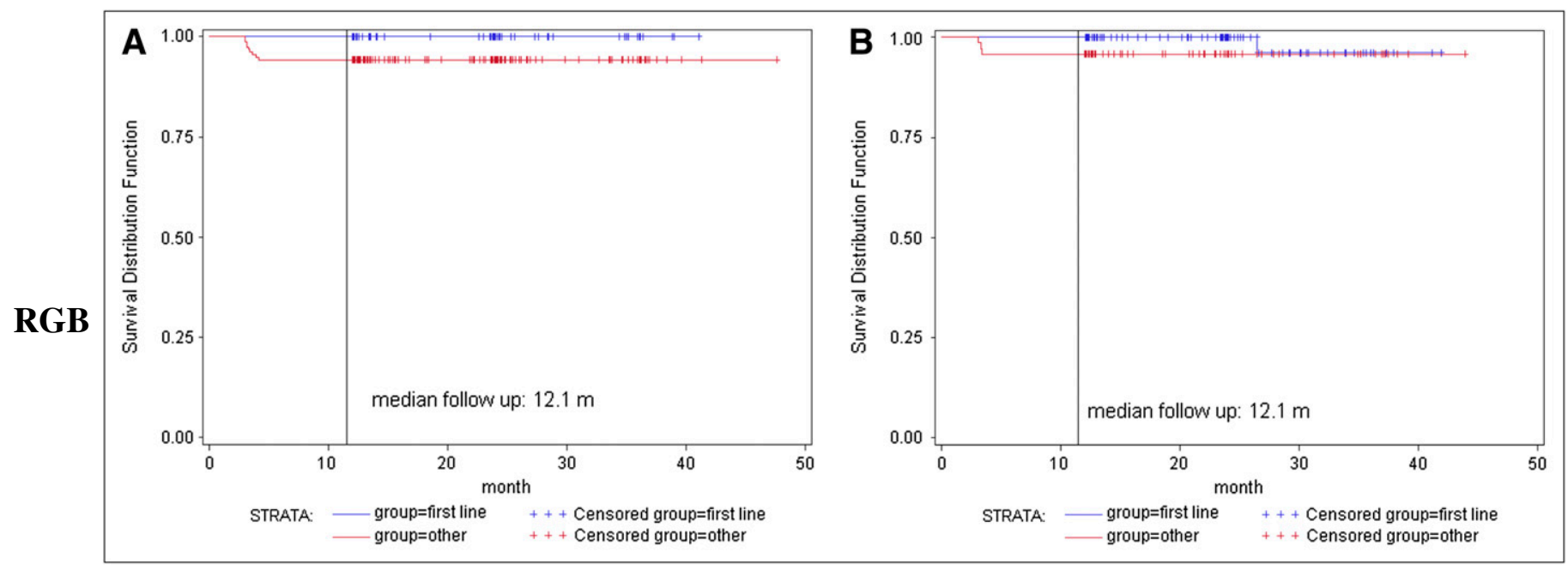

FIGURE 2. Time to progression according to indication (first line, consolidation after first-line and primary therapy vs. recurrence and therapy refractory, other) for patients with $\mathrm{FL}$ (log-rank test, $P=0.052)(\mathrm{A})$ and with other lymphoma subtypes (log-rank test, $P=0.18)(\mathrm{B})$.

treated relapsed or refractory patients $(8)$ or rituximabrefractory patients (16) with FL, CR rates (30\% and 15\%) and overall response rate $(80 \%$ and $74 \%)$ were lower than with our collective, with a CR rate of $73 \%$ for patients with FL. For the FL patients, PFS in the pivotal studies was 6.811.2 mo, which is shorter than in our collective, with a mean PFS of 33.6 mo. Adverse events were primarily hematologic and were comparable with the data in our collective, with grade 4 neutropenia and thrombocytopenia and grade 2 anemia after radioimmunotherapy.

The difference in CR rates and PFS in the pivotal studies and in the RIT-N study is most probably due to a different patient selection in clinical practice after the registration of the drug. In clinical practice, patients registered in the RIT$\mathrm{N}$ receive radioimmunotherapy earlier in their treatment course than in the pivotal studies. In the RIT-N, $45 \%$ of patients with FL and 69\% of patients with other lymphoma subtypes received radioimmunotherapy as consolidation therapy after first- or second-line treatments, whereas most patients in the pivotal studies received a median of 4 previous lymphoma treatments (range, 1-9) and had in 1 study (16) rituximab-refractory disease. In this survey, radioimmunotherapy was used earlier in the disease course by physicians in their everyday clinical practice.

These results may explain the better CR rates in the RIT$\mathrm{N}$ study, with a CR rate of $75 \%$, compared with $15 \%-30 \%$ in the pivotal studies. In the RIT-N study, $52.9 \%$ of the patients with FL are treated with radioimmunotherapy as first- and second-line therapy; because it is well known that $\mathrm{CR}$ rates decrease with each relapse or treatment line in FL, this observation leads to a better CR rate and time to progression rate, compared with patients treated later in the treatment course with radioimmunotherapy (Table 2; Fig. 3 ). In addition, radioimmunotherapy as consolidation after first-line therapy for FL can lead to a conversion rate from partial remission to CR in $77 \%$ of the patients in the FIT study (14). However, tumor stage at the time of radioimmunotherapy is not documented for patients with secondand third-line therapy in the RIT-N study and might also differ significantly from the pivotal studies. Because outcome may be better in lower stages at the time of relapse, this possible bias may contribute to this finding.

The fact that radioimmunotherapy is being used earlier in the treatment course in a large number of patients from around the world who were recruited to this registry analysis reflects the conviction of lymphoma physicians that radioimmunotherapy is a safe and feasible treatment option, especially for patients with FL but also for patients with other lymphoma subtypes, with an acceptable toxicity.

Of special interest is the observation that radioimmunotherapy results are particularly good, with high response rates and a long PFS in both FL and the other lymphoma subtypes, when radioimmunotherapy is used as part of the first-line treatment - for example, in consolidation to eliminate residual disease. Hopefully these results will lead to new randomized controlled studies of radiotherapy as firstline treatment that include modern first-line options such as

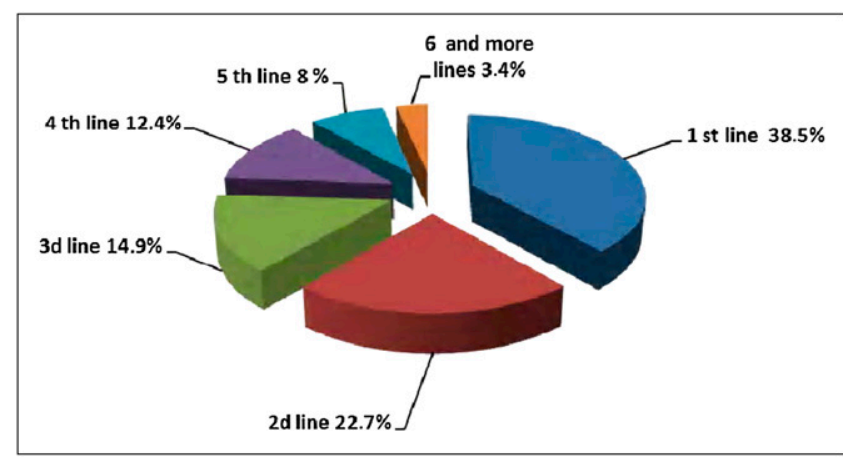

RGB

FIGURE 3. Use of radioimmunotherapy by line of treatment. 

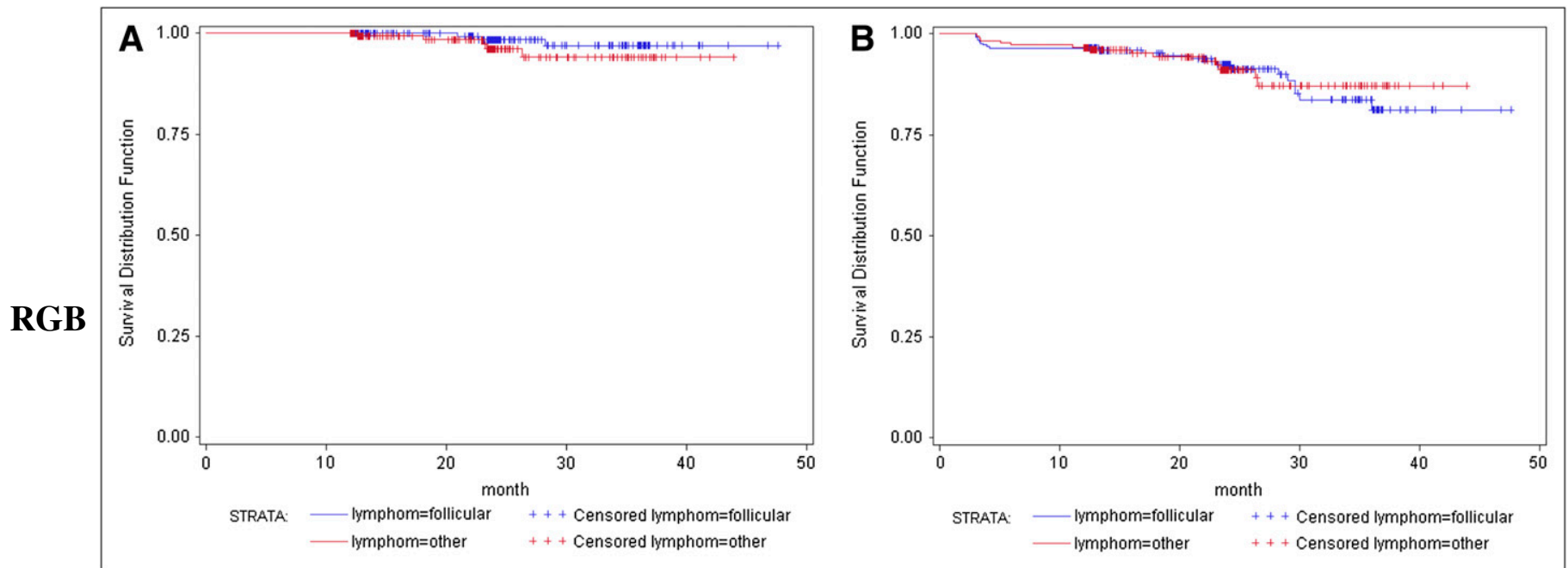

FIGURE 4. OS (A) and PFS (B) according to lymphoma subtype.

those with rituximab and may thereby confirm the results obtained by Morschhauser et al. (14).

With regard to the excellent result for the $42 \%$ of patients with other lymphoma subtypes treated outside the approved indication, our analysis demonstrates-as was previously shown (17) - that radioimmunotherapy is a feasible and good treatment option, with a safe and low-toxicity profile in lymphomas other than FL, the labeled indication.

\section{CONCLUSION}

Registry data are-in comparison to data obtained from prospective clinical trials-limited by the heterogeneity of patients' inclusion criteria, ill-defined response criteria, unavailability of certain data, and incorrect submission of data, because monitoring of source data cannot be performed. However, our results, which include large numbers of patients treated with radioimmunotherapy, give an unbiased picture of clinical practice with radioimmunotherapy and prove that even outside the labeled indication, radioimmunotherapy is safe and effective. These promising results should lead to controlled trials that may better define the optimal role of radioimmunotherapy in the treatment algorithm of lymphoma patients. Also, subgroups (e.g., marginal zone, mantle cell lymphoma) that might especially benefit from radioimmunotherapy need to be defined by these trials. Data from this registry could provide design strategies for clinical trials in applications beyond FL and

TABLE 4

Hematotoxicity After Radioimmunotherapy

\begin{tabular}{lccccc}
\hline & \multicolumn{2}{c}{ FL } & & \multicolumn{2}{c}{ Other lymphoma } \\
\cline { 2 - 3 } \cline { 5 - 6 } Parameter & $n$ & Median nadir & & $n$ & Median nadir \\
\hline Hemoglobin & 243 & $10.8 \mathrm{~g} / \mathrm{dL}$ & & 173 & $9.7 \mathrm{~g} / \mathrm{dL}$ \\
Leukocytes & 179 & $2.0 \mu \mathrm{L}$ & & 129 & $2.2 \mu \mathrm{L}$ \\
Platelets & 245 & $194 \mu \mathrm{L}$ & & 174 & $13.000 \mu \mathrm{L}$ \\
\hline
\end{tabular}

can also be used as a basis for pharmacoeconomic analyses. Radioimmunotherapy is safe, with tolerable side effects, and efficacious in the treatment of not only FL but also many other subtypes of B-cell lymphoma. On an individual basis, physicians prefer this treatment option over other available options. To extend the registration of this treatment modality, prospective trials are urgently needed.

\section{DISCLOSURE STATEMENT}

The costs of publication of this article were defrayed in part by the payment of page charges. Therefore, and solely to indicate this fact, this article is hereby marked "advertisement" in accordance with 18 USC section 1734.

\section{ACKNOWLEDGMENT}

We acknowledge the support of Michael Lorsbach, CRO, Alcedis GmbH; Francesco D'Amore, National Chairman Denmark; Didier Decaudin, National Chairman France; and Rod Johnson, National Chairman U.K. The RIT-N is sponsored by Bayer Health Care. No other potential conflict of interest relevant to this article was reported.

\section{REFERENCES}

1. Ahmed S, Winter JN, Gordon LI, Evens AM. Radioimmunotherapy for the treatment of non-Hodgkin lymphoma: current status and future applications. Leuk Lymphoma. 2010;51:1163-1177.

2. Heinzelmann F, Engelhard M, Ottinger H. Follicular lymphoma: the role of radiotherapy for stages I and II. Strahlenther Onkol. 2010;186:191-196.

3. Liu Q, Fayad L, Cabanillas F. Improvement of overall and failure free survival in stage IV follicular lymphoma: 25 years of treatment experience at the University of Texas MD Anderson Cancer Center. J Clin Oncol. 2006;24:1582-1589.

4. Fischer RI, LeBlanc M, Press OW. New treatment options have changed the survival of patients with follicular lymphoma. J Clin Oncol. 2005;23:84478452 .

5. Gallagher CJ, Gregory WM, Jones AE. Follicular lymphoma: prognostic factors for response and survival. J Clin Oncol. 1986;4:1470-1480.

6. Johnson PW, Rohatiner AZ, Whelan JS. Patterns of survival in patients with recurrent follicular lymphoma: a 20 year study from a single center. $J$ Clin Oncol. 1995;13:140-147. 
7. Connors JM. Radioimmunotherapy: hot new treatment for lymphoma. $N$ Engl J Med. 2005;352:496-498.

8. Witzig TE, Gordon LI, Cabanillas F. Randomized controlled trial of yttrium-90labeled ibritumumab tuxetan radioimmunotherapy versus rituximab immunotherapy for patients with relapsed or refractory low-grade, follicular, or transformed B-cell non-Hodgkin's lymphoma. J Clin Oncol. 2002;20:2453-2463.

9. Gopal AK, Gooley TA, Maloney DG. High dose radioimmunotherapy versus conventional high dose therapy and autologous hematopoietic stem cell transplantation for relapsed follicular non-Hodgkin lymphoma: a multivariable cohort analysis. Blood. 2003;102:2351-2357.

10. Kaminski MS, Estes J, Zasadny KR. Radioimmunotherapy with iodine ${ }^{131} \mathrm{I}$ tositumomab for relapsed or refractory B-cell non-Hodgkin lymphoma: updated results and long term follow up of the University of Michigan experience. Blood. 2000;96:1259-1266.

11. Fisher RI, Kaminski MS, Wahl RL. Tositumomab and iodine-131 tositumomab produces durable complete remissions in a subset of heavily pretreated patients with low-grade and transformed non-Hodgkin's lymphomas. J Clin Oncol. 2005;23:7565-7573.
12. Witzig TE, Molina A, Gordon LI. Long term responses in patients with recurring or refractory B-cell non-Hodgkin lymphoma treated with yttrium 90 ibritumomab tiuxetan. Cancer. 2007;109:1804-1810.

13. Mark S. Kaminski, M.D., Melissa Tuck, M.A. ${ }^{131}$ I-tositumomab therapy as initial treatment for follicular lymphoma. N Engl J Med. 2005;352:441-449.

14. Morschhauser F, Radford J, Van Hoof A. Phase III trial of consolidation therapy with yttrium-90-ibritumomab tiuxetan compared with no additional therapy after first remission in advanced follicular lymphoma. J Clin Oncol. 2008;26:51565164 .

15. Dreyling M, Trümper L, von Schilling C. Results of a national consensus workshop: therapeutic algorithm in patients with follicular lymphoma-role of radioimmunotherapy. Ann Hematol. 2007;86:81-87.

16. Witzig TE, Flinn IW, Gordon LI. Treatment with ibritumomab tiutexan radioimmunotherapy in patients with rituximab refractory follicular non-Hodgkin's lymphoma. J Clin Oncol. 2002;20:3262-3269.

17. Morschhauser F, Illidge T, Huglo D. Efficacy and safety of yttrium-90 ibritumomab tiuxetan in patients with relapsed or refractory diffuse large B-cell lymphoma not appropriate for autologous stem-cell transplantation. Blood. 2007; 110:54-58. 